\title{
Effect of Annealing on the Morphology, Structure and Photoelectric Properties of AZO/Pt/FTO Trilayer Films
}

\author{
Li-Jing Huang $\cdot$ Nai-Fei Ren $\cdot$ Bao-Jia Li $\cdot$ Ming Zhou
}

Received: 9 April 2014/Revised: 30 July 2014/Published online: 7 January 2015

(c) The Chinese Society for Metals and Springer-Verlag Berlin Heidelberg 2015

\begin{abstract}
Aluminum-doped zinc oxide/platinum/fluorine-doped tin oxide (AZO/Pt/FTO) trilayer films were prepared by sputtering 5-nm-thick Pt layers and 150-nm-thick AZO layers in sequence on commercial FTO glass. The effects of onestep annealing and layer-by-layer annealing on the morphology, structure and photoelectric properties of the AZO/Pt/FTO trilayer films were comparatively analyzed. It is found that the both annealing approaches increased the grain size and improved the crystallinity of the films, leading to enhancement in transmittance and conductivity. However, layer-by-layer annealing led to the formation of quasi-continuous or continuous AZO layers, different from the sparsely distributed AZO particles brought about by one-step annealing, resulting in excellent optical and electrical properties. Specifically, after layer-by-layer annealing at $400{ }^{\circ} \mathrm{C}$ for both $\mathrm{Pt}$ and $\mathrm{AZO}$ layers, the AZO/Pt/FTO trilayer film showed an increase in average transmittance from $71.3 \%$ to $85.3 \%$ and a decrease in sheet resistance from 7.5 to $5.6 \Omega / \square$, leading to the highest figure of merit of $3.64 \times 10^{-2} \Omega^{-1}$.
\end{abstract}

KEY WORDS: Annealing; Magnetron sputtering; Optical property; Electrical property; AZO/Pt/FTO trilayer film

\section{Introduction}

Transparent conducting oxide (TCO) films, which are widely used in photoelectric device applications such as thin-film

Available online at http://link.springer.com/journal/40195

L.-J. Huang · B.-J. Li · M. Zhou

School of Materials Science and Engineering, Jiangsu

University, Zhenjiang 212013, China

e-mail: lij_huang@126.com

L.-J. Huang · N.-F. Ren · B.-J. Li · M. Zhou

Jiangsu Provincial Key Laboratory of Center for Photon

Manufacturing Science and Technology, Jiangsu University,

Zhenjiang 212013, China

N.-F. Ren $(\bowtie)$

School of Mechanical Engineering, Jiangsu University,

Zhenjiang 212013, China

e-mail: rnf_ujs@126.com solar cells [1], flat-panel displays [2], gas sensors [3] and organic light-emitting diodes [4], have attracted tremendous academic and industrial interests in recent years. Among TCO films, tin-doped indium oxide (ITO) [5, 6], fluorine-doped tin oxide (FTO) [6] and aluminum-doped zinc oxide (AZO) [7] single-layer films hold greater promise for potential applications in solar cells because of their excellent optical and electrical properties. However, with the development of thinfilm solar cells, there is an increasing requirement for the photoelectric property of single-layer TCO films. To meet this requirement, new multilayer composite films have been constantly developed for improving transparency and conductivity of single-layer films [8-10]. Many multilayer films, such as ITO/Ag/ITO [11], ITO/Pt/ITO [12], AZO/Ag/AZO [13, 14], AZO/Au/AZO [15], AZO/Al/AZO [16] and $\mathrm{ZnO} /$ $\mathrm{Cu} / \mathrm{ZnO}[17,18]$, have been prepared and display high transparency and low resistivity. It is noteworthy that $\mathrm{Pt}$ possesses the virtues of high conductivity and excellent stability, enabling it to act as an effective interlayer material for 
multilayer film electrodes in thin-film solar cells especially in thin-film dye-sensitized solar cells [19]. Despite the high material cost of $\mathrm{Pt}$, using small amount of Pt to form a very thin Pt interlayer (several nm in thickness) can reduce the cost of the multilayer film [20].

It is well known that annealing treatment is also a conventional and effective technique to improve the photoelectric properties of TCO films. Annealing can improve crystallinity of the films through promoting grain growth and recrystallization, which will significantly affect the electrical and optical properties of TCO films [21, 22]. Tong et al. [23] reported that the electrical resistivity of AZO films annealed in hydrogen at $300{ }^{\circ} \mathrm{C}$ decreased from $9.8 \times 10^{-4}$ to $3.5 \times 10^{-4} \Omega \mathrm{cm}$. Sheu et al. [24] found that the resistivity of gallium-doped $\mathrm{ZnO}(\mathrm{GZO})$ films reduced from $1.4 \times 10^{-1}$ to $5.3 \times 10^{-4} \Omega \mathrm{cm}$ after the films being annealed in nitrogen under the temperatures ranging from 400 to $800{ }^{\circ} \mathrm{C}$. There are already many reports about one-step annealing approach for enhancing the photoelectric properties of multilayer TCO films [25, 26], but relatively few studies have focused on layer-by-layer annealing approach.

In this study, Pt layers and AZO layers were deposited in sequence by direct current (DC) magnetron sputtering on commercial FTO glass. Two strategies, i.e., one-step annealing and layer-by-layer annealing, were adopted to improve the photoelectric properties of the as-deposited AZO/Pt/FTO trilayer films. The effect of annealing approach on the morphology, structure and performance of the films was investigated, based on which the more effective annealing approach with the optimum annealing temperature was proposed.

\section{Experimental}

\subsection{Pre-treatment of FTO Glass}

The commercial FTO glass was cut into small pieces of $20 \mathrm{~mm} \times 20 \mathrm{~mm}$ and cleaned with deionized water, acetone and anhydrous ethanol in an ultrasonic bath each for $10 \mathrm{~min}$, followed by blowing high-purity (99.99\%) nitrogen to dry the film surfaces.

\subsection{Layer-by-Layer Annealing of AZO/Pt/FTO Films}

Firstly, 5-nm-thick Pt layer was deposited on the pre-cleaned FTO glass by DC magnetron sputtering coater (Emitech $\mathrm{K} 575 \mathrm{X}$ ) at room temperature using a metallic $\mathrm{Pt}$ target (99.99\% purity). The deposition chamber was evacuated to a base pressure of about $1 \times 10^{-3} \mathrm{~Pa}$, and then high-purity (99.99\%) argon was introduced. The working pressure and current were $35 \mathrm{~Pa}$ and $60 \mathrm{~mA}$, respectively. The ultimate thicknesses of the Pt layers were monitored in situ by a quartz crystal thickness monitor. After that, the obtained Pt/FTO bilayer films were thermal annealed at $400{ }^{\circ} \mathrm{C}$ in a tube furnace (Hefei Risine CVD(Z)-06/60/3). During annealing, high-purity $(99.99 \%)$ nitrogen with a constant flow rate of $10 \mathrm{~mL} / \mathrm{min}$ was introduced into the furnace chamber and maintained for $20 \mathrm{~min}$. Subsequently, 150-nm-thick AZO layer was deposited on the annealed Pt/FTO film by the $\mathrm{K} 575 \mathrm{X}$ coater using a zinc-aluminum alloy target (2 wt $\%$ $\mathrm{Al}_{2} \mathrm{O}_{3}$ ). The working current used here was $150 \mathrm{~mA}$, and the other details of deposition process were the same as described above for deposition of Pt layers. Then, the as-deposited AZO/Pt/FTO trilayer film was annealed in the tube furnace at a certain temperature $\left(300,400\right.$ or $\left.500{ }^{\circ} \mathrm{C}\right)$ for $20 \mathrm{~min}$ with a nitrogen flow rate of $10 \mathrm{~mL} / \mathrm{min}$.

\subsection{One-Step Annealing of AZO/Pt/FTO Films}

One-step annealing of AZO/Pt/FTO trilayer film was also carried out for the purpose of comparison. Firstly, 5-nmthick Pt layer and 150-nm-thick AZO layer were deposited in sequence on the pre-cleaned FTO glass by the K575X coater at room temperature using the metallic Pt target and zinc-aluminum alloy target, respectively. All the details of deposition process were the same as those of the AZO/Pt/ FTO film prepared by layer-by-layer annealing. Then, the as-deposited AZO/Pt/FTO trilayer film was annealed in the tube furnace at a certain temperature $\left(300,400\right.$ or $\left.500{ }^{\circ} \mathrm{C}\right)$ for $20 \mathrm{~min}$ with a nitrogen flow rate of $10 \mathrm{~mL} / \mathrm{min}$.

\subsection{Characterization}

The morphologies and thicknesses of the films were observed by a scanning electron microscope (SEM) (Carl Zeiss EVO MA10). The particle/grain size distributions and average particle/grain sizes on the film surfaces were obtained by Nano measure software. The 3D images with root-mean-square (RMS) roughnesses were scanned with an atomic force microscope (AFM) (Asylum Research MFP-3D-SA). The crystal structures of the films were examined by an X-ray diffractometer (XRD) (Rigaku $\mathrm{D} / \mathrm{max} 2500 \mathrm{VB} 3+/ \mathrm{PC})$ using $\mathrm{Cu} K \alpha$ radiation $(\lambda=0.1541 \mathrm{~nm})$ in the scan range of $2 \theta$ between $20^{\circ}$ and $80^{\circ}$. The optical properties were measured by a spectrophotometer (Shimadzu UV2550). The electrical properties were evaluated via a digital fourpoint probe instrument (Suzhou Baishen SX1944) and a Hall effect measurement system (Accent HL5500PC).

\section{Results and Discussion}

\subsection{Surface Morphology of AZO/Pt/FTO Films}

Figure 1 shows the surface morphology of the as-deposited AZO/Pt/FTO trilayer film. The inset in Fig. 1a 
provides the particle/grain size distributions on the film surface, and the average particle/grain size was taken as the quantity to compare the grain sizes for this and subsequent films [27]. The film had a rough surface and exhibited some AZO particles with an average size of $\sim 160 \mathrm{~nm}$, as shown in Fig. 1a. From the cross-sectional view shown in Fig. 1b, it is observed that the FTO layer exhibits the columnar growth of pyramidal shapes with a thickness of about $750 \mathrm{~nm}$, and the monitored thicknesses of the Pt and AZO layers (5 and $150 \mathrm{~nm}$, respectively) can be further verified.

Figure 2 shows the surface morphologies of the annealed AZO/Pt/FTO trilayer films at 300, 400 and $500{ }^{\circ} \mathrm{C}$, respectively. The insets display the particle/grain size distributions on the corresponding film surfaces. After one-step annealing, the films were covered by sparsely distributed AZO particles rather than continuous AZO layers (Fig. 2a-c). It is unfavorable for the enhancement of the film quality, although the average particle size of AZO layer (250, 270 and $300 \mathrm{~nm}$ in Fig. 2a-c, respectively) becomes greater than that of the as-deposited AZO/Pt/FTO film $(\sim 160 \mathrm{~nm})$. Furthermore, the film prepared by onestep annealing at $500{ }^{\circ} \mathrm{C}$ showed some nonuniformly distributed agglomerations consisting of small particles and displayed a broad particle size distribution (Fig. 2c), which is detrimental to the optical property of the film as will be confirmed later in transmittance analysis. In the case of the layer-by-layer-annealed AZO/Pt/FTO films (Fig. 2d, e), quasi-continuous or continuous AZO layers containing densely distributed particles were obtained. Especially at $400{ }^{\circ} \mathrm{C}$ (Fig. 2e), the average particles size $(\sim 280 \mathrm{~nm})$ became greater and the particles distributed more uniform than that of the as-deposited AZO/Pt/FTO film. It may be attributed to the effective improvement in crystallinity of the film, which will be discussed later. Uniform and larger particles were also obtained on film surfaces through layerby-layer annealing in other reports [28]. However, when the annealing temperature increased to $500{ }^{\circ} \mathrm{C}$, agglomerated particles with a nonuniform distribution were observed on the film surface, resulting in an average particle size of $\sim 320 \mathrm{~nm}$ (Fig. 2f). Obviously, annealing temperature was a crucial parameter that can affect the surface morphology of the films.

The 3D AFM images with a scanned region of $5 \mu \mathrm{m} \times 5 \mu \mathrm{m}$ for the as-deposited AZO/Pt/FTO film and the AZO/Pt/FTO films annealed at $400{ }^{\circ} \mathrm{C}$ by the two approaches are displayed in Fig. 3. The as-deposited film exhibited a rough surface with nonuniformly distributed pyramidal particles (Fig. 3a). The surfaces of the annealed films seemed rougher and consisted of obviously enlarged particles with relative smooth edges (Fig. 3b, c). It is notable that the particles on the surface of the layer-by-layer-annealed film are larger and more densely distributed as compared to those on that of the one-step-annealed one, which is consistent with the above SEM analysis. Figure 4 gives the surface RMS roughnesses of all the films extracted from AFM data. The asdeposited AZO/Pt/FTO film had a RMS roughness of $20.13 \mathrm{~nm}$. Both the one-step-annealed and layer-by-layerannealed films showed a similar trend of variation in RMS roughness. Specifically, with increasing annealing temperature from 300 to $500{ }^{\circ} \mathrm{C}$, the RMS roughnesses of the onestep-annealed and layer-by-layer-annealed films increased from 21.89 to 32.57 and 26.69 to $35.85 \mathrm{~nm}$, respectively. $\mathrm{Ng}$ et al. [29] reported that when sol-gel AZO films were annealed at various temperatures from 100 to $400{ }^{\circ} \mathrm{C}$, their RMS roughness also increased from 12 to $50 \mathrm{~nm}$. The increase in RMS roughness may be ascribed to the grain growth and improved crystallinity [29, 30].
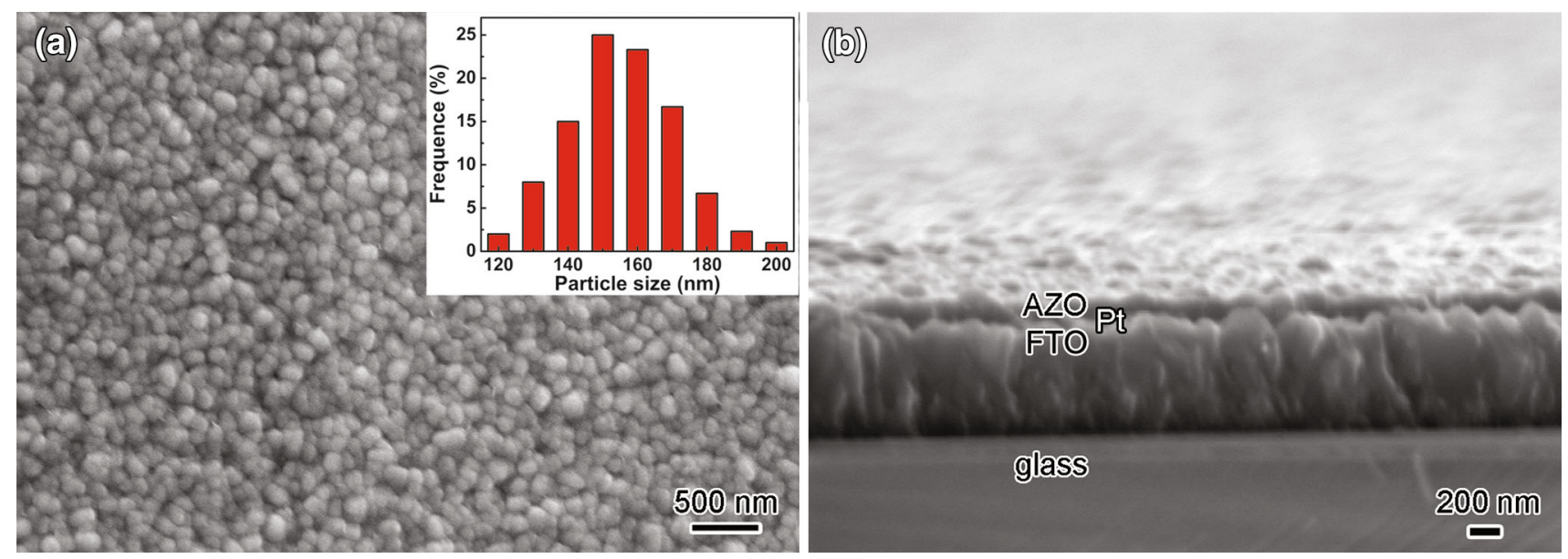

Fig. 1 SEM images of the as-deposited AZO/Pt/FTO film: a top view (the inset shows the particle size distribution on the film surface); $\mathbf{b}$ crosssectional view 

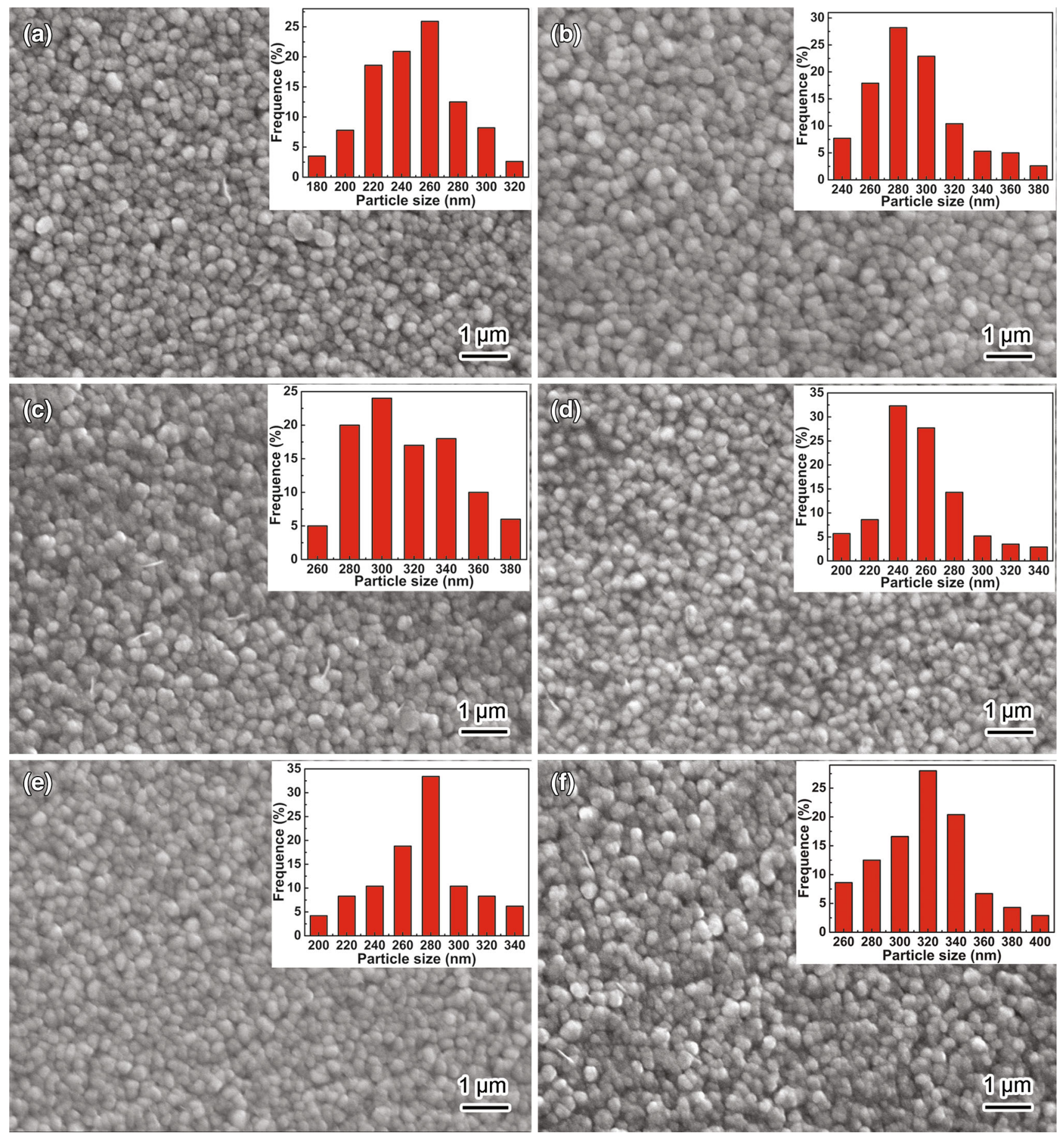

Fig. 2 SEM images of a-c the one-step-annealed, $\mathbf{d}-\mathbf{f}$ the layer-by-layer-annealed AZO/Pt/FTO films at various temperatures: a, $\mathbf{d} 300{ }^{\circ} \mathrm{C} ; \mathbf{b}$, e $400{ }^{\circ} \mathrm{C}$; c, f $500{ }^{\circ} \mathrm{C}$. The insets show the particle size distributions on the film surfaces

\subsection{Structural Characterization of AZO/Pt/FTO Films}

Figure 5a shows the XRD patterns of the as-deposited $\mathrm{AZO} / \mathrm{Pt} / \mathrm{FTO}$ trilayer film and the films obtained by layerby-layer annealing under the temperatures ranging from 300 to $500{ }^{\circ} \mathrm{C}$. It can be noticed that all the films exhibit the indexed diffraction peaks that are consistent with the standard values of the $\mathrm{SnO}_{2}$ tetragonal structure (JCPDS no. 41-1445), the Pt face-centered cubic structure (JCPDS no. 04-0802) and the $\mathrm{ZnO}$ hexagonal wurtzite structure (JCPDS no. 36-1451). The strongest intensity in the $\mathrm{SnO}_{2}$ (200) diffraction peak confirms the columnar growth of the 
(a)

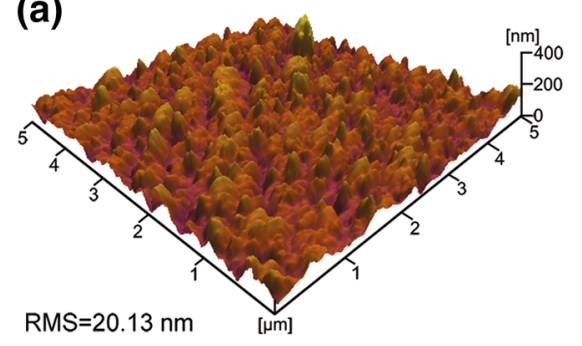

(b)

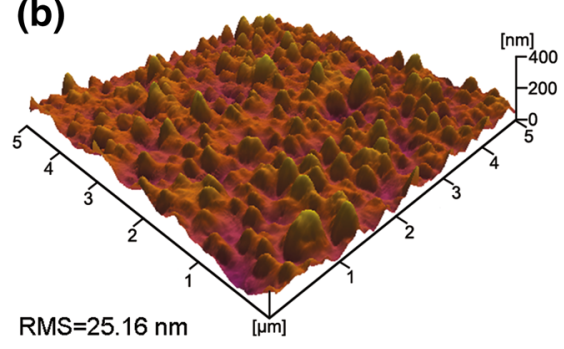

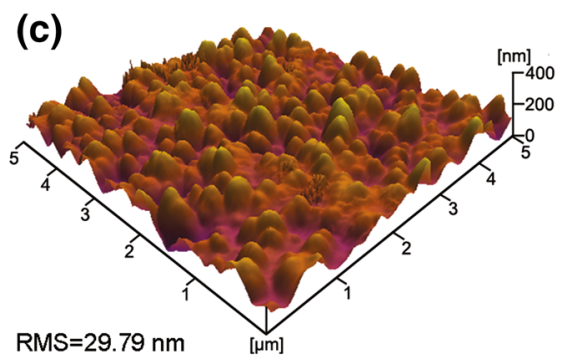

Fig. 3 AFM images of the as-deposited AZO/Pt/FTO film a, the one-step-annealed AZO/Pt/FTO film at $400{ }^{\circ} \mathrm{C}$ b, the layer-by-layer-annealed $\mathrm{AZO} / \mathrm{Pt} / \mathrm{FTO}$ film at $400{ }^{\circ} \mathrm{C}$ c

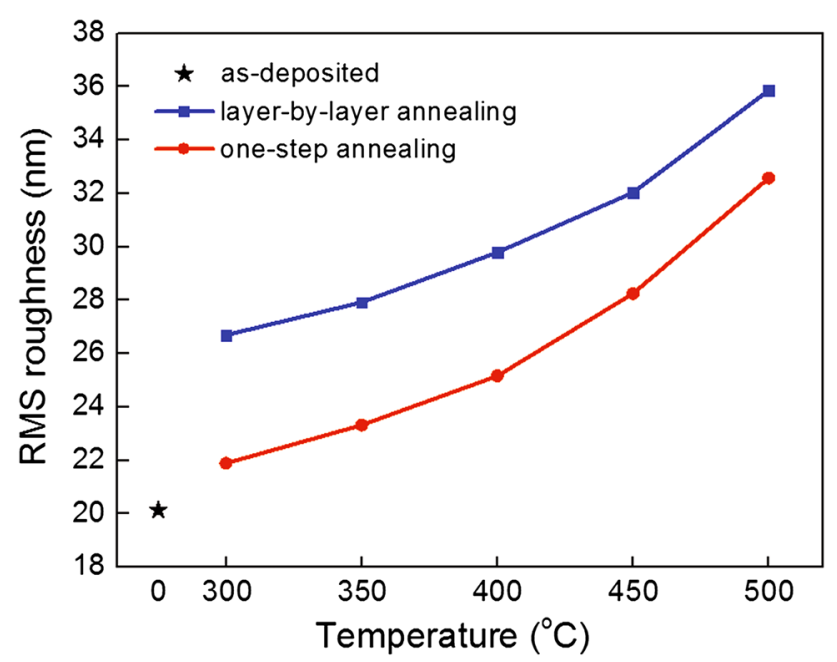

Fig. 4 Extracted surface RMS roughnesses of the as-deposited and the annealed $\mathrm{AZO} / \mathrm{Pt} / \mathrm{FTO}$ trilayer films

FTO layer [31], which has been indicated in the section of SEM analysis. Due to the annealing effect on the $\mathrm{Pt}$ and AZO layers, the Pt (111) peaks as well as the $\mathrm{ZnO}$ (100),
(002) and (101) peaks of all the layer-by-layer-annealed films, as compared to those of the as-deposited film, showed slightly higher intensity and lower full width at half maximum (FWHM), indicating that crystal defects in the $\mathrm{Pt}$ and AZO layers were effectively reduced [32]. Moreover, with annealing temperature increasing from 300 to $500{ }^{\circ} \mathrm{C}$, the $\mathrm{ZnO}$ (002) peaks showed an increase in the intensity and a decrease in the FWHM as a result of a gradual improvement in crystallinity of the films [33].

Figure $5 \mathrm{~b}$ presents the XRD patterns of the as-deposited AZO/Pt/FTO trilayer film and the films annealed at $400{ }^{\circ} \mathrm{C}$ by layer-by-layer annealing and one-step annealing. It is found that the annealed films exhibit stronger $\mathrm{SnO}_{2}$ (200) peaks and $\mathrm{ZnO}$ (002) peaks corresponding an improvement in crystallinity of the as-deposited films. Only the Pt layer in the layer-by-layer-annealed AZO/Pt/FTO film underwent direct annealing, resulting in relatively higher intensity and lower FWHM of the Pt (111) peak. In addition, compared with the as-deposited and one-step-annealed films, the $\mathrm{SnO}_{2}$ (200) peak of the layer-by-layer-annealed film were relatively stronger and narrower, indicating higher crystallinity of the film and fewer defects in the film
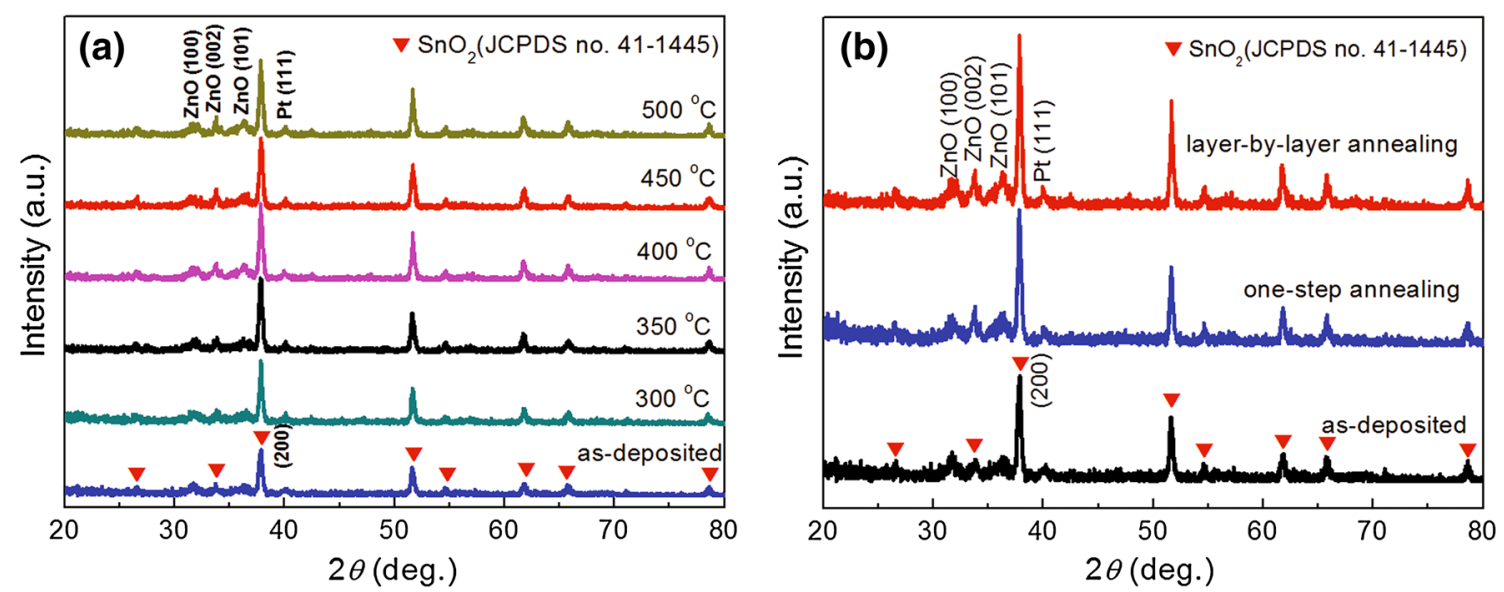

Fig. 5 a XRD patterns of the as-deposited AZO/Pt/FTO trilayer film and the layer-by-layer-annealed AZO/Pt/FTO trilayer films at various temperatures; b XRD patterns of the as-deposited AZO/Pt/FTO trilayer film and the AZO/Pt/FTO trilayer films annealed at $400{ }^{\circ} \mathrm{C}$ by two approaches 
[32]. The twice annealing of the FTO layer in the layer-bylayer annealing process should be responsible for this result.

\subsection{Photoelectric Properties of AZO/Pt/FTO Films}

Figure 6a shows transmittance spectra of the as-deposited AZO/Ag/FTO trilayer film and the films annealed at different temperatures. All the films exhibited a drop-off in transmittance at shorter wavelengths that was related to the fundamental absorption caused by band-to-band transition [34]. The transmittance of the as-deposited AZO/Ag/FTO film was significantly improved after employing both layer-by-layer annealing and one-step annealing. But the increment achieved by layer-by-layer annealing was obviously greater than that by one-step annealing, which may be ascribed to the fact that the quasi-continuous or continuous AZO layer can suppress the total internal reflection in the Pt and FTO layers $[35,36]$. It is noteworthy that the absorption edges for all the annealed films shifted to the shorter wavelength side compared with the as-deposited one, and the wavelengths of the absorption edges for the layer-by-layer-annealed films decreased with an increase in annealing temperature, similar to the results for AZO films reported by Ali et al. [34]. According to previous researches, the blueshift of absorption edge should be due to the improved crystallinity and the increased carrier concentration (called as Burstein-Moss effect) [37, 38]. Figure 7 presents the dependence of the carrier concentration of all the AZO/Ag/FTO trilayer films on annealing temperature. It is seen that the carrier concentrations of both the onestep-annealed and layer-by-layer-annealed films are higher than that of the as-deposited one, and increase obviously with increasing annealing temperature. These provide evidence for the above explanation.

The average optical transmittance in the wavelength range of 400-1,000 $\mathrm{nm}$ of the films is illustrated in Fig. 6b.
The average transmittance of the as-deposited AZO/Pt/ FTO trilayer film was about $71.3 \%$. After layer-by-layer annealing, the average transmittance greatly increased from $80.2 \%$ to $85.3 \%$ with increasing annealing temperature from 300 to $400{ }^{\circ} \mathrm{C}$, and then dropped to $82.6 \%$ with increasing annealing temperature to $500{ }^{\circ} \mathrm{C}$. For the onestep-annealed AZO/Pt/FTO trilayer films, the average transmittance increased to a maximum of $77.3 \%$ at an annealing temperature of $400{ }^{\circ} \mathrm{C}$ and then gently decreased to $75.3 \%$ with increasing annealing temperature to $500{ }^{\circ} \mathrm{C}$. No matter what annealing methods and annealing temperatures are adopted, the increase in particle/grain size and the improvement in crystallinity lead to less grain boundaries and reduce the light scattering at the grain boundaries, resulting in the enhancement of the transmittance [39]. The decrease in average transmittance of the films that annealed at a temperature higher than $400{ }^{\circ} \mathrm{C}$ may be related to the nonuniformly distributed agglomerated particles that were observed on the film surfaces (Fig. 2c, f), since the nonuniformity of particles/grains can bring about the increases

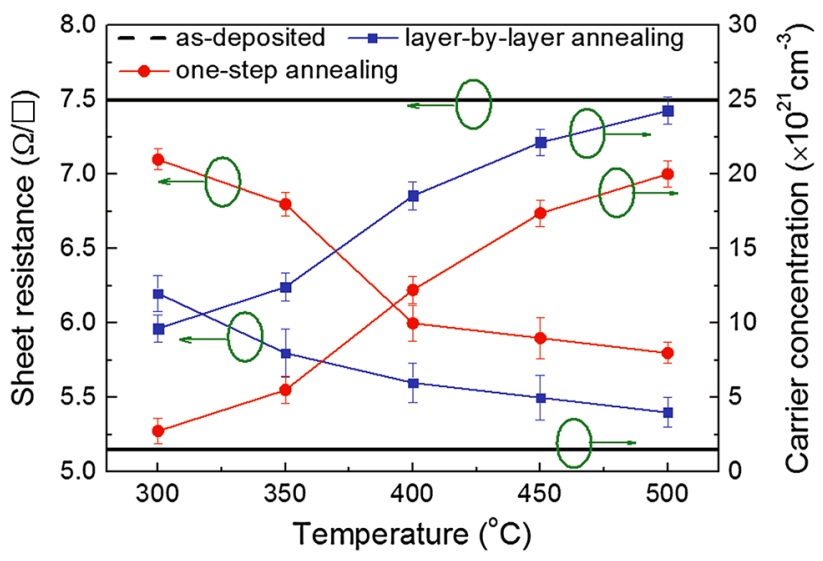

Fig. 7 Sheet resistances and carrier concentrations of the as-deposited and the annealed AZO/Pt/FTO trilayer films
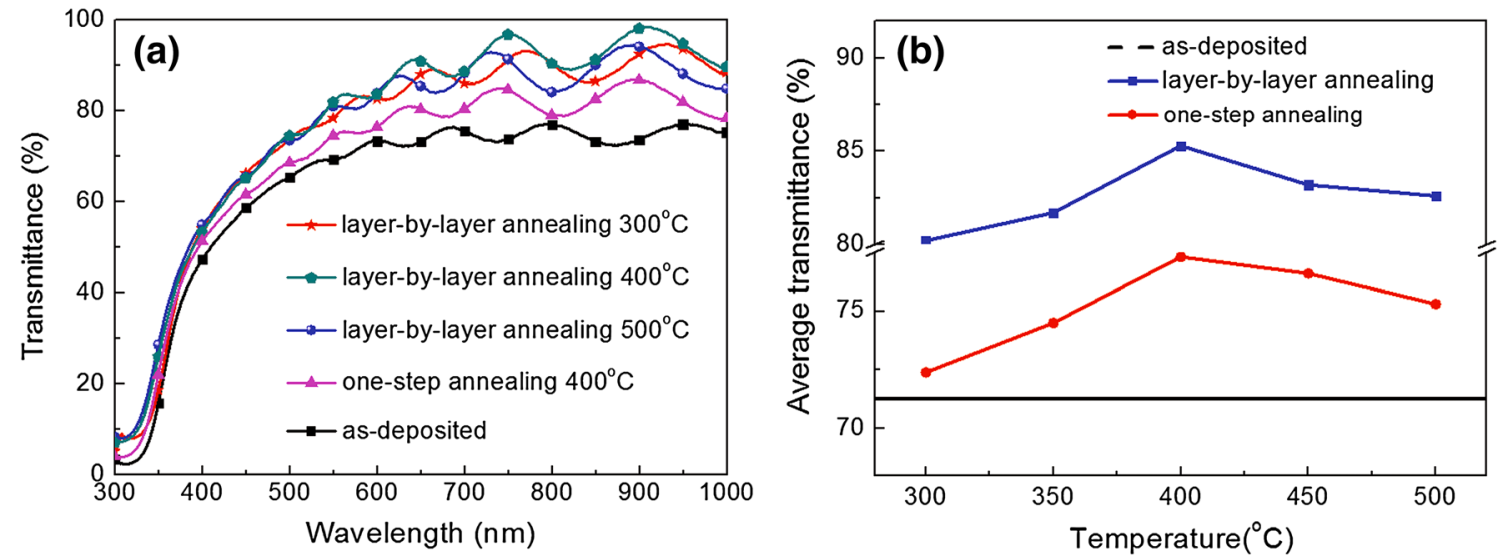

Fig. 6 a Transmittance spectra and $\mathbf{b}$ average transmittances $(400-1,000 \mathrm{~nm})$ of the as-deposited and the annealed AZO/Pt/FTO trilayer films 
in scattering and absorption of light in the film, and therefore, reduce the transparency of the film $[40,41]$.

The dependence of sheet resistance on the annealing temperature for the annealed AZO/Pt/FTO trilayer films is also shown in Fig. 7. Compared with that of the as-deposited AZO/ $\mathrm{Pt} / \mathrm{FTO}$ film $(7.5 \Omega / \square)$, the sheet resistances of all the annealed AZO/Pt/FTO trilayer films were relatively low. Annealing does not only eliminate parts of the crystal defects in the films (as indicated in XRD analysis), it also enhances carrier mobility and reduces carrier scattering at the grain boundaries by increasing grain size [42, 43]. All these should be responsible for the improvement in conductivity of the films. The sheet resistance of the one-step-annealed films decreased from 7.1 to $5.8 \Omega / \square$ with increasing annealing temperature from 300 to $500{ }^{\circ} \mathrm{C}$. The sheet resistance of the layer-by-layer-annealed films decreased from 6.2 to $5.4 \Omega / \square$ with the same change in annealing temperature. However, at the same annealing temperature, the sheet resistance of the film prepared by layer-by-layer annealing was obviously lower than that of by one-step annealing (e.g., 5.6 vs. $6.0 \Omega / \square$, at $400{ }^{\circ} \mathrm{C}$ ), which may be attributed to the greater grain size and fewer defects in the former film. In addition, the blueshift of absorption edge in the transmittance spectra (Fig. 6a) that corresponds to the increase in carrier concentration may be conducive to improving conductivity of the films [44], which can further explain this result.

The quality of the trilayer films can be determined by a figure of merit $\left(F_{\mathrm{TC}}\right)$ defined as $F_{\mathrm{TC}}=T^{10} / R_{\mathrm{S}}$ [45], where $T$ is the average transmittance in the wavelength range of $400-1,000 \mathrm{~nm}$ and $R_{\mathrm{S}}$ is the sheet resistance. The figures of merit of the as-deposited and annealed AZO/Pt/FTO trilayer films in our work are shown in Fig. 8. The figure of merit of the as-deposited film was only $0.45 \times 10^{-2} \Omega^{-1}$ and was enhanced by both layer-by-layer and one-step annealing. The film prepared by layer-by-layer annealing at $400{ }^{\circ} \mathrm{C}$ had the highest figure of merit of $3.64 \times 10^{-2} \Omega^{-1}$, almost three times higher than that of the corresponding one-stepannealed film $\left(1.27 \times 10^{-2} \Omega^{-1}\right)$. By adopting the same definition for $T$, Dimopoulos et al. [15] calculated $F_{\mathrm{TC}}$ for $\mathrm{AZO} / \mathrm{Au} / \mathrm{AZO}$ trilayer films and obtained the best value of $6.9 \times 10^{-3} \Omega^{-1}$, significantly lower than that is presented here. Sutthana et al. [14] and Lin et al. [16] took the average transmittance in the visible range as $T$, yielding the highest $F_{\mathrm{TC}}$ of $6.9 \times 10^{-4} \Omega^{-1}$ for $\mathrm{AZO} / \mathrm{Ag} / \mathrm{AZO}$ films and $1.94 \times 10^{-2} \Omega^{-1}$ for $\mathrm{AZO} / \mathrm{Al} / \mathrm{AZO}$ films, respectively. These are uncomparable to the corresponding value of $2.42 \times 10^{-2} \Omega^{-1}$ for the AZO/Pt/FTO film in this study. In addition, Sahu and Huang [18] used the maximum $T$ value for the calculation of $F_{\mathrm{TC}}$ for $\mathrm{ZnO} / \mathrm{Cu} / \mathrm{ZnO}$ multilayer film. In comparison, the highest $F_{\mathrm{TC}}$ calculated by using the maximum $T$ in our work is $15.31 \times 10^{-2} \Omega^{-1}$ and outperforms their result $\left(1.97 \times 10^{-2} \Omega^{-1}\right)$. Our work indicates that layer-by-layer annealing, rather than one-step annealing, is

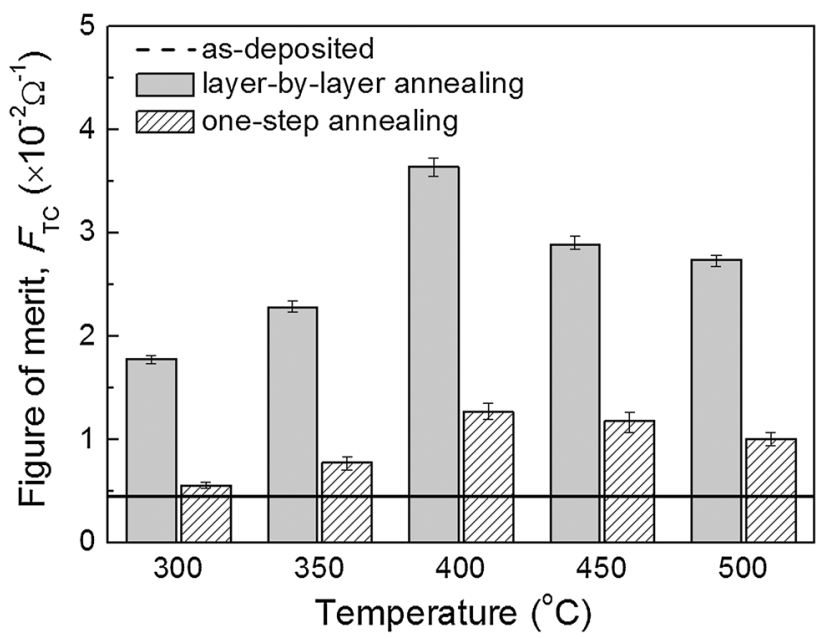

Fig. 8 Figures of merit of the as-deposited and the annealed AZO/Pt/ FTO trilayer films

more effective for improving the quality of multilayer films. More importantly, the AZO/Pt/FTO trilayer films with higher quality obtained by layer-by-layer annealing are quite valuable for window electrodes in various photovoltaic applications particularly in dye-sensitized solar cells.

\section{Conclusions}

In summary, AZO/Pt/FTO trilayer films were prepared through coating commercial FTO glass with 5-nm-thick Pt layers and 150-nm-thick AZO layers by DC magnetron sputtering. The as-formed AZO/Pt/FTO trilayer films, which possessed an average transmittance of $71.3 \%$, a sheet resistance of $7.5 \Omega / \square$ and a figure of merit of $0.45 \times 10^{-2} \Omega^{-1}$, were treated by one-step annealing and layer-by-layer annealing. In the present work, all the annealed films exhibited an increase in the grain size and an improvement in the crystallinity, thereby achieving the enhancement in photoelectric properties. However, onestep annealing led to the formation of sparsely distributed AZO particles, resulting in relatively smaller increment in transmittance and conductivity. The layer-by-layerannealed films were covered with quasi-continuous or continuous AZO layers and exhibited excellent optical and electrical properties. In particular, the film treated by layerby-layer annealing at $400{ }^{\circ} \mathrm{C}$ had the highest figure of merit of $3.64 \times 10^{-2} \Omega^{-1}$ with an average transmittance of $85.3 \%$ and a sheet resistance of $5.6 \Omega / \square$. The results indicate that layer-by-layer annealing can more effectively improve the photoelectric properties of multilayer films.

Acknowledgments This work was supported by the National Key Basic Research Development Program of China (No. 2011CB013000), the Jiangsu Province Research Innovation Program 
of College Graduate (No. CXZZ13_0663), the Senior Talent Research Foundation of Jiangsu University (No. 13JDG045) and the Open Research Fund Program of Jiangsu Provincial Key Laboratory of Center for Photon Manufacturing Science and Technology (No. GZ201307).

\section{References}

[1] S. Ito, T.N. Murakami, P. Liska, C. Grätzel, M.K. Nazeeruddin, M. Grätze, Thin Solid Films 516, 4613 (2008)

[2] G.S. Chae, Jpn. J. Appl. Phys. 40, 1282 (2001)

[3] T. Miyata, T. Hikosaka, T. Minami, Sens. Actuators, B 69, 16 (2000)

[4] S.B. Kang, J.W. Lim, S. Lee, J.J. Kim, H.K. Kim, J. Phys. D 45, 325102 (2012)

[5] S.I. Na, S.S. Kim, J. Jo, D.Y. Kim, Adv. Mater. 20, 4061 (2008)

[6] S. Cornelia, G. Constantin, A. Stefan, Thin Solid Films 519, 595 (2010)

[7] J.W. Leem, D.H. Joo, J.S. Yu, Sol. Energy Mater. Sol. Cells 95, $2221(2011)$

[8] A. Zyoud, I. Saa'deddin, S. Khudruj, Z.M. Hawash, D.H. Park, G. Campet, H.S. Hilal, Solid State Sci. 18, 83 (2013)

[9] J.C. Berne'de, L. Cattin, M. Morsli, Y. Berredjem, Sol. Energy Mater. Sol. Cells 92, 1508 (2008)

[10] D.S. Ghosh, T.L. Chen, N. Formica, J. Hwang, I. Bruder, V. Pruneri, Sol. Energy Mater. Sol. Cells 107, 338 (2012)

[11] K.H. Choi, J.Y. Kim, Y.S. Lee, H.J. Kim, Thin Solid Films 341, 152 (1999)

[12] J.I. Choi, J.Y. Lee, J.H. Park, J.H. Chae, H.J. Park, D. Kim, J. Phys. Chem. Solids 70, 272 (2009)

[13] F.S. Li, Y.Z. Zhang, C.X. Wu, Z.X. Lin, B.B. Zhang, T.L. Guo, Vacuum 86, 1895 (2012)

[14] S. Sutthana, N. Hongsith, S. Choopun, Curr. Appl. Phys. 10, 813 (2010)

[15] T. Dimopoulos, G.Z. Radnoczi, B. Pécz, H. Brückl, Thin Solid Films 519, 1470 (2010)

[16] Y.S. Lin, W.C. Tseng, J. Electron. Mater. 41, 437 (2012)

[17] K. Sivaramakrishnan, T.L. Alford, Appl. Phys. Lett. 94, 052104 (2009)

[18] D.R. Sahu, J.L. Huang, Appl. Surf. Sci. 253, 827 (2006)

[19] H. Kim, G. Veerappan, J.H. Park, Electrochim. Acta 137, 164 (2014)

[20] C.C. Yang, H.Q. Zhang, Y.R. Zheng, Curr. Appl. Phys. 11, S147 (2011)

[21] B.L. Zhu, X.H. Sun, X.Z. Zhao, F.H. Su, G.H. Li, X.G. Wu, J. Wu, R. Wu, J. Liu, Vacuum 82, 495 (2008)
[22] C. Guillén, J. Herrero, Vacuum 84, 924 (2010)

[23] H. Tong, Z.H. Deng, Z.H. Liu, C.G. Huang, J.Q. Huang, H. Lan, C. Wang, Y.G. Cao, Appl. Surf. Sci. 257, 4906 (2011)

[24] J.K. Sheu, K.W. Shu, M.L. Lee, C.J. Tun, G.C. Chi, J. Electrochem. Soc. 154, 521 (2007)

[25] H. Kermani, H.R. Fallah, M. Hajimahmoodzadeh, Phys. E 47, 303 (2013)

[26] A. Kim, Y. Won, K. Woo, C.H. Kim, J. Moon, ACS Nano 7, $1081(2013)$

[27] Y. Yang, P. Lan, M. Wang, T. Wei, R. Tan, W. Song, Nanoscale Res. Lett. 7, 481-1 (2012)

[28] H.C. Wang, C.H. Liao, Y.L. Chue, C.C. Lai, P.C. Chou, S.Y. Ting, Opt. Mater. Express 3, 295 (2013)

[29] Z.N. Ng, K.Y. Chan, T. Tohsophon, Appl. Surf. Sci. 258, 9604 (2012)

[30] H.M. Lee, Y.J. Lee, I.S. Kim, M.S. Kang, S.B. Heo, Y.S. Kim, D. Kim, Vacuum 86, 1494 (2012)

[31] C. Agashe, J. Hüpkes, G. Schöpe, M. Berginski, Sol. Energy Mater. Sol. Cells 93, 1256 (2009)

[32] L.J. Huang, N.F. Ren, B.J. Li, M. Zhou, Mater. Lett. 116, 405 (2014)

[33] Z.Z. Zhi, Y.C. Liu, B.S. Li, X.T. Zhang, Y.M. Lu, D.Z. Shen, X.W. Fan, J. Phys. D 36, 719 (2003)

[34] H.M. Ali, M.M. Abd El-Raheem, N.M. Megahed, H.A. Mohamed, J. Phys. Chem. Solids 67, 1823 (2006)

[35] J.H. Lim, K.H. Lee, D.C. Lim, J. Korean Phys. Soc. 57, 1229 (2010)

[36] N.F. Ren, L.J. Huang, M. Zhou, B.J. Li, Ceram. Int. 40, 8693 (2014)

[37] S. Suzuki, T. Miyata, M. Ishii, T. Minami, Thin Solid Films 434, $14(2003)$

[38] H. Kim, J.S. Horwitz, S.B. Qadri, D.B. Chrisey, Thin Solid Films 420-421, 107 (2002)

[39] R.J. Winfield, L.H.K. Koh, S. O’Brien, G.M. Crean, Appl. Surf. Sci. 254, 855 (2007)

[40] M. Hezam, N. Tabet, A. Mekki, Thin Solid Films 518, 161 (2010)

[41] B.R. Kumar, T.S. Rao, J. Optoelectron. Biomed. Mater. 4, 35 (2012)

[42] S. Lee, J. Seong, D.Y. Kim, J. Korean Phys. Soc. 56, 782 (2010)

[43] B.J. Li, L.J. Huang, M. Zhou, N.F. Ren, B. Wu, Ceram. Int. 40, 1627 (2014)

[44] G. Fang, D. Li, B.L. Yao, Vacuum 68, 363 (2002)

[45] M.S. Kim, D.Y. Kim, M.Y. Cho, G. Nam, S. Kim, D.Y. Lee, S.O. Kim, J.Y. Leem, Vacuum 86, 1373 (2012) 